\title{
Constancy and illusion of apparent direction of rotary motion in depth: Tests of a theory*
}

\author{
R. P. POWER \\ The Queen's University of Belfast, Belfast Bt7 INN, Northern Ireland \\ and \\ R. H. DAY \\ Monash University, Clayton 3168, Victoria, Australia
}

\begin{abstract}
An explanation of apparent direction of rotary motion in depth derived from a general theory of perceptual constancy and illusion is proposed with experimental data in its support. Apparent direction of movement is conceived of as exhibiting perceptual constancy or illusion as a function of apparent direction of orientation in depth for plane objects and apparent relative depth for three-dimensional objects. Apparent reversals of movement direction represent either regular fluctuations between constancy and illusion of direction as a function of valid and invalid stimuli for orientation, or irregular and random fluctuations in their absence. In three preliminary experiments, the apparent movement direction of plane ellipses was investigated as a function of surface pattern information for orientation, and in Experiment I apparent reversals during 20-revolution trials were studied. In Experiment II, apparent movement direction of $3 D$ elliptical $V$ shapes as a function of surface pattern information for relative depth was investigated. In addition to supporting the explanation proposed, the data offer a resolution of a conflict between different theories of apparent reversal of motion in depth.
\end{abstract}

Objects rotating in depth relative to an observer are frequently reported as reversing their apparent direction of motion (Kenyon, 1898; Miles, 1929; Ames, 1951), an effect sometimes called the Ames illusion. Day and Power (1965) critically examined explanations of these reversais proposed by Ames (1951), Pastore (1952), and Graham (1963) and concluded that none were entirely satisfactory, mainly because they were concerned to explain apparent reversals (ARs) of asymmetrical objects such as trapezoids. whereas it had been shown that symmetrical circular and elliptical objects and irregular plane objects also exhibit reversals (Day \& Power, 1963. 1965). However, an alternative explanation put forward by Day and Power (1965) also proved unsatisfactory and has had to be rejected. It was originally proposed that the patterns of motion at the retina for clockwise $(\mathrm{CW})$ and counterclockwise (CCW) movement were identical and therefore ambiguous as stimuli or "cues"1 for motion direction. Since. in Day and Power's experiments, ARs occurred on about half the occasions on which the plane object passed through the frontoparallel plane. it was argued that this ambiguity resulted in chance fluctuations in apparent direction and consequently of apparent orientation. That is, apparent orientation was held to be a consequence of apparent direction, a view opposite to that of earlier explanations. This theory had to be abandoned in the light of two serious objections. First. the assumption of identical retinal motion patterns for $\mathrm{CW}$ and $\mathrm{CCW}$ movement.

\footnotetext{
*Requests for reprints should be addressed to R. P. Power. Department of Psychology. The Queen's University of Belfast. Belfast BT7 INN. Northern Ireland.
}

while true for parallel projections, is not so for polar projections such as oscur at the eye (Hershberger, 1967). Identity for polar projections would effectively occur only when the angle subtended at the eye by the rotating object was so small as to render the difference in relative velocities of symmetrically disposed points between $\mathrm{CW}$ and $\mathrm{CCW}$ movement below threshold (Börjesson, 1971; Hershberger, 1967; MacRae \& Power. 1972). Second, unknown at the time to Day and Power. Zegers (1964) had shown that frequency of AR decreases with visual angle (as would be expected from the previous point). It became clear that the visual angle subtended by the plane objects used by Day and Power $(1963,1965)$ happened to be that resulting in ARs on about half the occasions on which the patterns passed through the frontoparallel plane.

\section{GENERAL CONSTANCY THEORY APPLIED TO APPARENT REVERSAL}

Apparent reversal of rotary motion in depth can be economically and satisfactorily explained in terms of a recently proposed theory of perceptual constancy and illusion (Day, 1972). Broadly, this theory asserts that illusions of such spatial properties as size, shape. orientation. and movement are consequent on the manipulation of stimuli which normally preserve perceptual constancy of those properties when their retinal projections vary. For example. size illusions occur when distance stimuli are manipulated and movement illusions occur when stimuli for head movement are varied. In the absence of constancy-preserving stimuli. the property being judged is perceptually unstable. so 
that fluctuations occur as a function of instructional, motivational, and chance factors. The theory can be easily applied to apparent direction of rotation in depth.

A plane object rotating in depth in one direction projects on the retina a movement pattern of variable direction: the total pattern is one of expansion and contraction as the object changes from the "edge-on" to the "full-on" position relative to $S$, and vice versa. However, even though the total movement pattern varies in its directional characteristics, the apparent direction of movement. $\mathrm{CW}$ or $\mathrm{CCW}$, is constant and veridical during normal binocular viewing of most objects. For such direction constancy to occur, valid information about the direction of orientation of the object in depth is necessary. For example, if the retinal image is undergoing contraction and the object is apparently and actually tilted with its near edge to the right of the far edge, its apparent direction must be CW. Similarly, if during retinal expansion the object is tilted with its near edge to the left of the far one, its apparent direction will also be $\mathrm{CW}$. Thus, continuous $\mathrm{CW}$ movement is dependent on valid information for the direction of object tilt during both the contraction and expansion phases of the retinal projection.

Information for object orientation in depth is provided by numerous stimuli, but if monocular observation is used to eliminate retinal disparity, and the viewing distance exceeds $1 \mathrm{~m}$, the distance within which convergence-accommodation is operative (Leibowitz \& Moore, 1966), three stimuli are critical: outline shape (Clark, Smith, \& Rabe, 1955), surface pattern and texture (Börjesson, 1971; Day \& Power, 1965), and motion perspective. For rotating objects, motion perspective derives from the relative angular velocity of surface points on either side of the rotation axis (Hershberger, 1967). It follows that if these three stimuli for orientation serve to maintain constancy of direction as the retinal projection expands and contracts, then their manipulation so as to change apparent direction of orientation from near edge to the right of far, to near edge to the left of far, or vice versa, must give rise to a change in the apparent direction of movement, i.e., to an illusion of movement direction. No such illusion will occur, of course, unless the modification of orientation stimuli is sufficient to induce a change in the direction of orientation, i.e., from tilt left to tilt right in depth relative to the observer. With such variation in orientation stimuli, the apparent direction of orientation of the object will be opposite to its true direction for half its rotation cycle, during which its apparent direction of movement must also be opposite to its true direction. For the other half of the cycle, constancy of movement direction will obtain. The ARs then represent changes from constancy to illusion of motion direction, since apparent orientation in depth determined by "valid" or "invalid" orientation stimuli changes regularly as the object passes from one direction of tilt to another.

In the absence of stimuli for orientation, as would be the case with an unpatterned textureless plane ellipse, ${ }^{2}$ fluctuations between the two directions of rotation unrelated to orientation would be expected to occur. Without the stabilizing effect of orientation stimuli, valid or invalid, the retinal expansion-contraction pattern is similar in principle to the ambiguous retinal projections of a stationary skeletal cube, the apparent form of which fluctuates (see Day, 1969). Instructional, motivational, and chance variables can be expected to exercise considerable influence under such conditions. Some effects of instructions have been demonstrated by Power (1965).

In this explanation, emphasis is shifted from ARs themselves to motion direction as a function of orientation information, with ARs being treated as points of change between these two perceptual states. In the complete absence of orientation stimuli, fluctuations between the two directions in an irregular pattern would be expected.

The strength of this explanation is that direction of rotary motion in depth can be viewed in the same context and explained in essentially the same terms as a wide range of perceptual constancies and their associated illusions (Day, 1972). The fact that there are only two directions of orientation in depth or two directions of movement does not affect the similarity in principle with other cases of constancy, illusion, and instability.

\section{TESTS OF THE GENERAL CONSTANCY THEORY}

If the theory outlined is valid, then both the apparent direction of movement and the frequency of ARs during a given number of revolutions can be predicted. Throughout the experiments reported here, only the surface pattern stimulus for orientation direction in depth was varied. The outline stimulus was eliminated by using elliptical objects. Motion perspective was minimized by using objects with a visual angle of less than $10 \mathrm{deg}$ (MacRae \& Power, 1972), rotating at relatively low $(4.75 \mathrm{rpm})$ angular velocity (Börjesson, 1971). Viewing throughout was monocular, thus eliminating binocular parallax. Observation distance was greater than $1 \mathrm{~m}$, thus reducing accommodationconvergence information (Leibowitz \& Moore, 1966).

The three preliminary experiments were designed to test the hypotheses that initial direction of rotary movement is a function of apparent direction of orientation as determined by surface pattern and that, in the absence of orientation stimuli, initial direction is random and independent of orientation. On the basis of these data, the first main experiment (Experiment I) examined the predictions that frequency of ARs varies with surface pattern information for orientation and that, in the absence of stimuli for orientation, ARs occur randomly. The explanation outlined applies not only to plane objects, but also to three-dimensional (3D) objects 
rotating in depth. Experiment II was concerned with the apparent direction of initial movement with 3D ubjects as a function of their "apparent" relative depth.

\section{APPARATUS}

Although the stimulus objects and procedures varied between experiments. the basic apparatus and conditions of observations were the same throughout and can conveniently be described first.

The stimulus objects were housed in a chamber $91 \mathrm{~cm}$ wide and high and $182 \mathrm{~cm}$ long. There were two viewing tubes, each of which gave a field of view 15 deg in diam. and an adjustable chinrest at one end of the chamber. A $40-\mathrm{W}$ circular fluorescent tube. $38 \mathrm{~cm}$ in outer diam, was mounted symmetrically about the viewing tubes inside the chamber so that the interior. including the stimulus object, was evenly illuminated with the source out of view. An aiternative method of illumination was an ultraviolet lamp placed below the circular fluorescent tube. Each of the two viewing tubes could be :losed by means of a lens cap. and a shutter could be raised or lowered by $E$ to expose or occlude the stimulus object at the beginning and end of a trial.

The stimulus objects were suspended trom a socket attached to the vertical drive shaft of a synchronous motor geared to rotate the object at $4.75 \mathrm{rpm}$. Once during each revolution, a cam on the drive shaft closed a switch which deflected one pen on a chart recorder. The remaining pen was activated by a press-switch operated by $\mathrm{S}$ to signal ARs. The axis of rotation was $132 \mathrm{~cm}$ from the outer ends of the viewing tubes, and the elliptical objects were so placed as to fall centrally in the field of view.

The elliptical plane objects (Fig. 1) used in the preliminary experiments and in Experiment I were cut from $1.2 \cdot \mathrm{mm}$ aluminum sheet. with a major axis of $22.5 \mathrm{~cm}$ ivisual angle: $9 \mathrm{deg} 36 \mathrm{~min})$ and a minor axis of $18 \mathrm{~cm}(7 \mathrm{deg} 36 \mathrm{~min}$. The 3D objects used in Experiment II were made from similar material and are described below.

For the preliminary experiments in which the orientation of the elliptical objects was judged. a plane rectangular object. $25.5 \mathrm{~cm}(9 \mathrm{deg} 36 \mathrm{~min}) \times 15 \mathrm{~cm}(6 \mathrm{deg} 18 \mathrm{~min})$ and cut from the same aluminum sheet as the ellipse. was mounted on an axis collinear with that of the ellipse above it. A regular rectangular pattern of $2.5-\mathrm{cm}$ blue and white bars was placed on the surface of the rectangle nearest to $\mathrm{S}$. Using a rotary control. $\mathrm{S}$ could adjust the orientation of the patterned rectangle to match that of the ellipse. The angle could be read from a protractor scale mounted concentrically about the axis on the floor of the chamber. Earlier experiments (Day \& Power. 1965) had shown that apparent slant could be easily and confidently matched by this means. Throughout all experiments, observations of both orientation and movement direction were made monocularly. using the preferred eve.

\section{PRELIMINARY EXPERIMENTS}

In each of the three preliminary experiments. separate groups of Ss first matched the apparent orientation of the frontoparallel ellipse. using the rectangular matching object. and then reported its apparent direction of movement as it rotated CS or CCW through 70 deg.

\section{Methods}

\section{Protiminam tixperiment I}

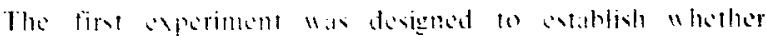
informatien for true diretion of ariontitton from surtiss

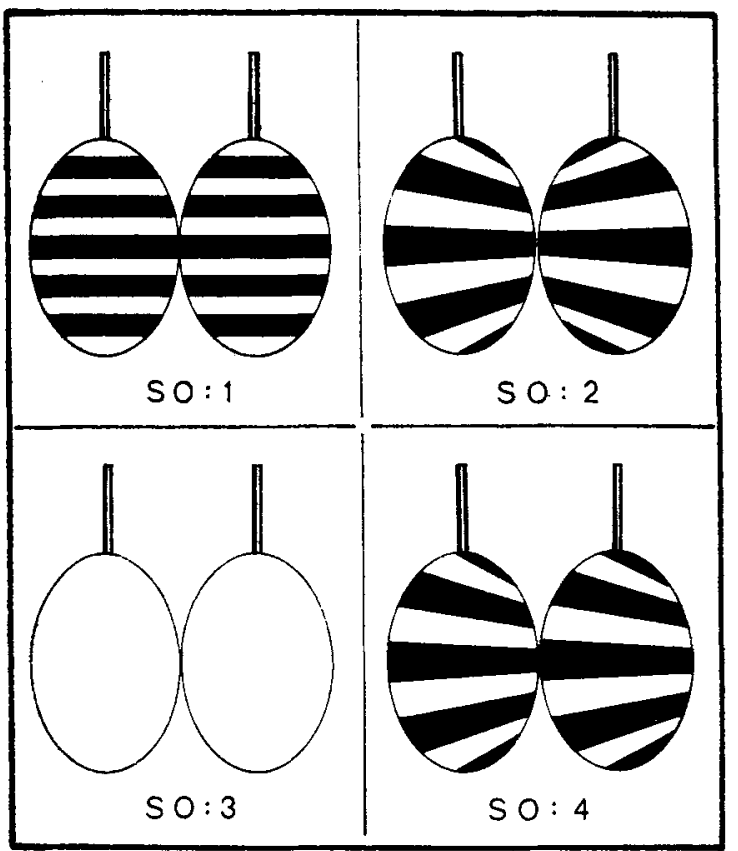

Fig. 1. Plane stimulus objects used in Experiment $\mathrm{I}$.

pattern results in veridical apparent direction. The elliptical object SO 1 . with a surface pattern of $2.5-\mathrm{cm}$ black and white bars (Fig. 1). was observed by 20 undergraduate Ss. After the frontoparallel ellipse had been matched for apparent orientation using the rectangle. it was rotated through 70 deg and $S$ reported apparent direction. The shutter was raised at the beginning of the orientation judgment phase and lowered after the ellipse had travelled through $70 \mathrm{deg}$. For half the group. movement direction was CW, and for the other half. CCW.

The mean apparent orientation of the frontoparallel (90-deg) ellipse was $90.6 \mathrm{deg}$, with a standard deviation of $3.3 \mathrm{deg}$. and 19 of the $20 \mathrm{Ss}$ correctly reported apparent direction. The single nonveridical report occurred for CCW movement.

\section{Preliminary Experiment?}

The procedures in the second experiment were the same as in the first. 20 Ss matching apparent orientation and judging movement direction of the stimulus object SO 2 (Fig. 1). The surface pattern of SO 2 was a 45 -deg transformation of that on SO 1. l or half the Ss. movement direction was Cll (broad end. of bars approaching). and for the other half. CCW.

The mean apparent orientation of the frontoparallel object was $58.6 \mathrm{deg}$. with the broad ends of the bars apparently nearer. The standard deviation was $23.6 \mathrm{deg}$. Of the $10 \mathrm{Ss}$ who observed Cll movement. apparent direction was veridical. whereas for the 10 who observed CCW movement. direction was nonveridical for 9 and veridical for 1 .

\section{Preliminary Experiment 3}

l sing the same procedurs as in the two earlier experiments. the aim of the third was to test the hypothesis that apparent dirsetion of movement in the absence of orientation stimuli is random and unrotated to surarent orientation. Sime the stimulu, object SO 3 (f ie. 1) wits elliptical. unpatterned. and viewed momocularls. information for orientation in depth was minimal. There nere 48 undereraduate $S$ s. 2t of whom observed

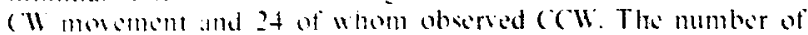


Ss was greater than in the previous experiment. since it was hoped to accept a null hypothesis that apparent orientation was not a determinant of apparent direction of movement.

The mean apparent orientation of SO 3 was $91.7 \mathrm{deg}$, with a standard deviation of $86.8 \mathrm{deg}$. The large standard deviation indicates considerable variation in apparent orientation. Of the $48 \mathrm{Ss}, 25$ gave veridical reports of apparent direction and 23 gave nonveridical reports. with the proportion of correct and incorrect reports about equal for the two directions.

\section{Conclusions from Preliminary Experiments}

Taken together, the data from the three preliminary experiments, requiring first a judgment of orientation followed by one of movement direction, strongly support the dependence of the second on the first for SO 1 and SO 2 and the independence of the two for SO 3. On contraction of the retinal image of SO 1 during either CW or CCW movement, apparent direction was veridical. Since it can be assumed that veridicality would also have obtained during retinal expansion, it can be concluded that stimulus information which results in veridical direction of orientation produces constancy of apparent direction of movement for the expanding-contracting retinal projection. In the case of SO 2 , the surface pattern resulted in a considerable discrepancy between actual and apparent orientation, with the broad ends of the converging pattern apparently near. As the retinal image contracted, therefore, movement direction was in accord with direction of apparent tilt. Therefore, apparent direction was veridical for one direction of motion but not for the other. Assuming that this would also be the case for the expansion phase, it can be concluded that for that part of the cycle in which there is correspondence between actual and apparent direction of orientation, constancy of movement direction would obtain, and for the other half, in which there is noncorrespondence, an illusion of movement direction. With minimization of orientation stimuli, as with SO 3, there was considerable variation in apparent orientation and apparent direction of movement, with no suggestion of the dependence of the second on the first.

In general, the data from the three preliminary experiments support the hypothesis that apparent direction of movement is determined by apparent direction of orientation and that, in the absence of stimuli for orientation, movement direction is unrelated to apparent orientation.

\section{EXPERIMENT I: REGULAR AND IRREGULAR APPARENT REVERSAL OF PLANE ELLIPSES}

The data from the three preliminary experiments suggest that since apparent direction of rotary motion is a function of the surface pattern stimulus for orientation, the frequency of ARs for a particular pattern during a set number of revolutions should be predictable. Thus. it would be expected that SO 1 would seldom, if ever. exhibit an AR. whereas SO 3 would do so on each occasion that the object passed through the frontoparallel plane. However, two considerations militate against this "strong" hypothesis about AR. First. during a complete revolution, an interposition stimulus for apparent orientation occurs as the "edge-on" orientation is reached in the median plane. It will be seen below that for certain arrangements of surface pattern this stimulus is important. Second, since careful measures had been taken to eliminate alternative stimuli for orientation, surface pattern was probably the only one available. It is conceivable that this single stimulus might not be sufficiently "strong" to control apparent orientation or movement direction completely. For these reasons, the first main experiment was designed to test the "weaker" hypothesis from the general theory that there would be a particular order of frequencies of AR for four conditions of surface pattern to be described.

\section{Method}

\section{Apparatus and Procedure}

The apparatus and general conditions of observation have already been described. The task required of $S$ was that of signaling each AR by operating the switch during a 20-revolution trial. Rotation began from the frontoparallel position, and the shutter was raised and lowered at the beginning and end of a trial. The instruction to Ss was to press the switch each time the object appeared to reverse its direction of motion. (See Power. 1967. for the complete instructions.) The objects in this experiment and in Experiment II were given a light coat of fluorescent paint and were illuminated by ultraviolet light. This meant that the rod supporting the object was not visible to the $S$ and could not, during the longer periods of observation. give information to true direction of rotation.

\section{Stimulus Objects and Predictions}

The four stimulus objects shown in Fig. 1 were used, SO 1, SO 2 , and SO 3 being those used in the three preliminary experiments. The fourth, SO 4, was the same as SO 3 except that the pattern of converging bars was reversed on one surface so that the broad ends of the bars on one corresponded with the narrow ends on the other.

Since constancy of movement direction occurred with SO 1, it was predicted that it would exhibit the lowest frequency of ARs. Furthermore, since apparent direction of SO 3 was random, a change in direction (i.e., an AR) could be expected on about half the occasions the surface passed through the frontoparallel plane, to give approximately $20 \mathrm{ARs}$ for a 20 -revolution trial. For SO 2, however, an AR was expected to occur regularly as the surface passed through the frontoparallel plane, with the total frequency approximating to 40 . The greatest frequency of ARs was expected for SO 4, since ARs were expected to occur occasionally in the median plane as well as regularly in the frontoparallel. The "back-to-front" arrangement of the pattern was such as to modify apparent direction of orientation as the object passed through the "edge-on" position. However, as already mentioned, because an interposition stimulus for orientation (one edge obscuring the other) also occurs at this stage of the cycle, the absolute frequency of ARs was uncertain. Thus, the expected order of frequency of ARs from lowest to 
highest for the four surface pattern conditions was SO 1. SO 3. SO 2. SO 4

\section{Subjects}

There were 96 Ss divided into four groups of 24 Ss each. One group was assigned to each condition. Half the $S s$ in each group observed $\mathrm{CW}$ movement and the other half, $\mathrm{CCW}$.

\section{Results and Discussion}

The mean frequencies of ARs together with standard deviations are shown for $\mathrm{CW}$ and $\mathrm{CCW}$ movement in Table 1. An analysis using the method of planned contrasts (Hays. 1963; Roger. 1965) showed that direction of rotation was a nonsignificant variable for each stimulus object. Each $F$ ratio was less than 1. It can be seen from Table 1 that the order of frequencies of $A R$ is the predicted one. An analysis using planned contrasts showed that mean frequency of $A R$ of each object differed from all others with $p<.05$. It can be noted also that the mean frequency of 12.9 for SO 1 is substantially higher than if constancy of direction had obtained throughout, i.e., a frequency of 0 . The occasional reversals are probably attributable to the presence of only one effective stimulus for orientation, that of surface pattern, rather than the multiple stimuli that are normally available. However. the frequencies of 21.6 and 36.3 for SO 3 and SO 2. respectively. accord well with expectancy (20 and 40 . respectively). ${ }^{3}$ In the case of SO 4, it is reasonable to suppose, as suggested earlier, that the strong interposition stimulus at the median orientation prevented the occurrence of more than a few ARs in addition to those in the frontoparallel position.

\section{EXPERIMENT II: APPARENT DIRECTION OF MOVEMENT OF THREE-DIMENSIONAL OBJECTS}

So far. almost all studies of AR of rotation in depth have involved plane objects. However, the explanation proposed here applies equally to $3 \mathrm{D}$ objects forming a $\mathrm{V}$ shape in depth (Fig. 2). In essentially the same way as perceptual constancy and illusion of apparent direction of movement in depth with plane objects is a function of apparent orientation. so constancy and illusion with $\mathrm{V}$

Table 1

Means and Standard Deviations of Frequency of Apparent Reversal for the Four Stimulus Objects Used in Experiment 1 (Fig. 1)

\begin{tabular}{llcccc}
\hline & & \multicolumn{4}{c}{ Stimulus Object } \\
\cline { 3 - 6 } Direction & Statistic & SO 1 & SO 2 & SO 3 & SO 4 \\
\hline \multirow{2}{*}{ CW } & Mein & 13.6 & 37.0 & 21.3 & 47.2 \\
& SD & 11.9 & 6.5 & 11.1 & 24.2 \\
(CII & Ms:in & 12.2 & 35.7 & 21.9 & 45.8 \\
& S1) & 7.3 & 5.6 & 11.4 & 21.11 \\
\hline
\end{tabular}

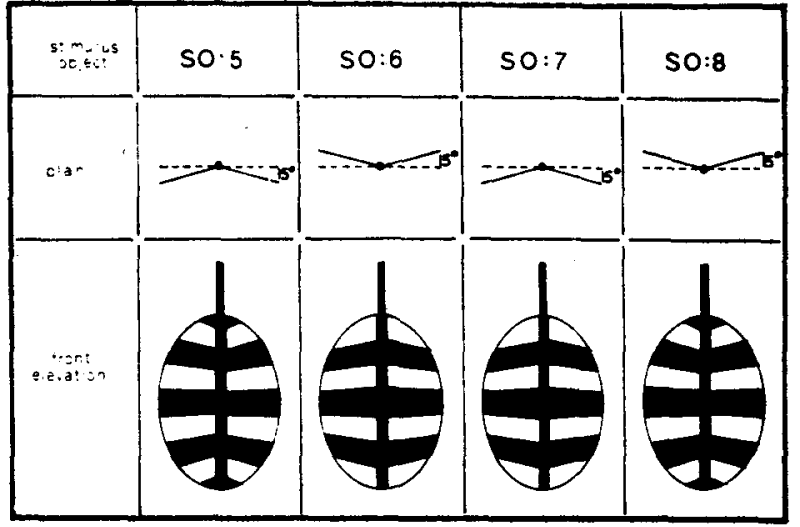

Fig. 2. Three-dimensional stimulus objects used in Experiment II.

objects is a function of apparent relative depth, i.e., relative depth of the apical ridge and edges.

When $\mathrm{V}$ objects such as those shown in Fig. 2 rotate from a position in which they are symmetrical about the median plane, one half of the retinal image contracts and the other expands. If expansion occurs on the left and contraction on the right with the apical ridge apparently near. the apparent direction of rotation must be $\mathrm{CCW}$, but if it occurs with the ridge apparently far, the apparent direction must be $\mathrm{CW}$. In brief, perceived direction of movement with a particular expansion-contraction pattern of the two halves of the image is a function of apparent depth. Thus. by systematically varying the surface pattern stimulus for depth, it should be possible to produce constancy and illusion of apparent direction. The purpose of Experiment II was to test the hypothesis that perceptual constancy and illusion of movement direction is a function of the apparent depth of $\mathrm{V}$ objects as determined by surface patterns. It can be noted that because of their depth and interposition stimuli for rotation direction, it is not possible to study ARs with these 3D objects.

\section{Method}

\section{Apparatus and Procedure}

The apparatus was that described above. At the beginning of a trial. the motor was switched on and the occluding shutter raised when the leading edye of the $\mathrm{V}$ was $10 \mathrm{deg}$ of rotation past the median plane. The shutter was closed when the trailing edge was approashing the median plane and $10 \mathrm{deg}$ from it. The total angle through which the object moved thus was 130 deg. At the conclusion of the observation. S was required to report apparent relative depth (ridge near and ridge far relative to the lateral cdges) and appirent direction of movement.

\section{Stimulus (Obiects and Predictions}

The stimulus objects SO 5. SO 6. SO 7. and SO . . shown in

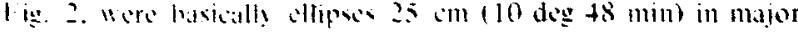
and 195 ind is deg +2 minl in minor ais but folded atong the 
Table 2

Frequency of Veridical (V) and Nonveridical (NV) Reports of Judged Shape and Veridical and Nonveridical Reports of Judged Motion Direction in Experiment II (Fig. 2)

\begin{tabular}{llrr} 
& & \multicolumn{2}{c}{ Judged Shape } \\
\cline { 3 - 4 } & & V & NV \\
\hline \multirow{2}{*}{ Judged Motion Direction } & V & 42 & 3 \\
& NV & 1 & 34 \\
\hline
\end{tabular}

major axis so that the inner angle was $150 \mathrm{deg}$. When symmetrical with reference to $S$ 's median plane, the minor axis subtended $8 \mathrm{deg} 24 \mathrm{~min}$ at the edge. The axis on which the objects were mounted was collinear with the central ridge. The surface patterns on SO 5 and SO 6 were 45 -deg transformations of parallel bars, $2.5 \mathrm{~cm}$ wide and with a convergence which corresponded with, and therefore enhanced, the actual relative depth of the object. It was predicted that for these objects constancy of apparent direction of motion would obtain, i.e., apparent direction would accord with actual direction. The patterns on SO 7 and SO 8 were similar but with a convergence pattern that did not correspond with the true relative depth of the objects. For these two, it was predicted that apparent direction of movement would be opposite to actual direction, i.e., an illusion of movement direction would occur.

\section{Subjects}

Four groups of 20 undergraduate Ss participated, one group per stimulus object. Half the $S$ s in each group observed $C W$ movement and the other half, CCW.

\section{Results and Discussion}

Apparent relative depth and apparent direction of rotation are shown for the four objects in Table 2 . Table 2 shows that there is a very consistent overall tendency to report either veridical relative depth and motion direction or nonveridical depth and motion direction. Thus, the overall results are convincing in demonstrating a close association between apparent relative depth and apparent direction of movement. For each of the eight conditions, 9 or 10 out of 10 Ss gave both responses as veridical or as nonveridical. The probability of obtaining such results under the null hypothesis is 0.022 (Siegel, 1956). For Objects SO 5 and SO 6, in which the perspective of the pattern corresponded with that of actual depth, 38 Ss correctly judged relative depth and veridically reported direction of movement. For SO 7 and SO 8 , on which the perspective was in noncorrespondence with the relative depth of the object, 38 Ss incorrectly judged relative depth (i.e., reported ridge far or near when, in fact, it was near and far, respectively) and gave nonveridical reports of apparent direction. It is reasonable to conclude that surface pattern determines apparent relative depth under the conditions reported and that apparent depth, in turn, determines apparent direction of rotary motion.

\section{DISCUSSION AND CONCLUSIONS}

Central to the theory proposed here is the contention that apparent direction of rotary movement in depth is a function of information for direction of object orientation in depth for plane objects and a function of relative depth for 3D objects. When in both cases stimuli result in correspondence between apparent and actual depth characteristics, apparent direction of movement is in accord with true direction, even though the directional features of the retinal image are variable. When stimuli for depth give rise to noncorrespondence between apparent and actual depth, apparent direction of movement is opposed to actual direction. From this standpoint, the much discussed ARs represent points of change between perceptual constancy and illusion of direction and in that sense are secondary effects. However, when depth information is much reduced or absent, ARs continue to occur, but irregularly, randomly, and unrelated to apparent orientation or shape in depth.

The data reported from the three preliminary experiments and first main experiment, using plane ellipses, and from the second experiment, using 3D ellipses, are generally in agreement with predictions from the theory. With plane objects, apparent direction was veridical with valid information for orientation in depth, nonveridical with invalid information, and unrelated to orientation and random in the absence of information with unpatterned objects. In Experiment $I$, the order of frequency of ARs for four conditions of pattern was as predicted, although ARs occurred when none were expected for one condition and occurred relatively infrequently for another. However, surface pattern was the only stimulus for orientation direction available, and it is reasonable to suppose that some random ARs would occasionally occur under such conditions. The infrequency of ARs in the median position is explainable in terms of the alternative stimulus of interposition for the direction of orientation depth. The data from Experiment II are clear in showing that the constancy/illusion argument applies also to 3D objects whose apparent depth characteristics control apparent direction of movement.

The explanation outlined here and the data in its support resolve an apparent conflict between the results of earlier experiments with rotating trapezoid shapes and those reported by Day and Power $(1963,1965)$ using symmetrical and irregular shapes. Whereas earlier theories (Ames, 1951; Graham, 1963; Pastore, 1952) and some recent formulations (Börjesson, 1971; Graham \& Gillam, 1970) have emphasized the role of apparent orientation in depth as the basis of regular ARs, Day and Power drew attention to irregular ARs occurring randomly and independently of apparent orientation. The formulations put forward by Börjesson and by Graham and Gillam are helpful, especially in correctly drawing attention to the role of apparent depth, which we had earlier discounted (Day \& Power, 1965), and the data reported here are not inconsistent with their theoretical positions. These positions, however, fall short 
of being comprohensive in that neither copes with ARs from unfamiliar irregular and elliptical shapes. The issue is resolved if apparent direction of movement. rather than ARs. is considered. Depending on stimulus information for orientation. apparent direction of movement will exhibit constancy or illusion and. if the information is appropriately manipulated. regular fluctuation botween the two will occur. However. if stimulus information for orientation in depth (or depth shape in the case of 3D objects) is lacking. apparent direction of motion is unstable and fluctuates irregularly.

Two further points can be usefully made in conclusion. First. surface pattern. as used in the experiments reported here, is one of numerous stimuli for apparent depth which. by itself. may not provide the most forceful or reliable information. It is likely that if other stimuli. such as shape and motion perspective. are manipulated together with pattern. apparent direction of movement and ARs could be predicted with considerable accuracy. Surface pattern has the advantage that it can be opposed on either side of a planar object. thus causing ARs in the median plane. Second, the explanation offered here is an aspect of a general theory of perceptual constancy and illusion (Day 1972). The present analysis shows that perceptual constancy, illusion. and instability apply to dichotomous variables, such as direction of movement. as well as to continuous variables. such as size and shape.

\section{REFERENCES}

Ames. A. Visual perception and the rotating trapezoidal window. Psychological Monographs. 1951.65(7. Whole No. 324).

Börjesson. E. Properties of changing patterns evoking visually perceived oscillation. Perception \& Psychophysics. 1971. 9. 303-308.

Clark. W. C., Smith. A. H., \& Rabe. A. The interaction of surface texture, outline gradient. and ground in the perception of slant. Canadian Journal of Psychology. 1956. 10. 1-8.

Day, R. H. Human perception. Sydney: Wiley. 1969.

Day. R. H. Visual spatial illusions: A general explanation. Science. 1972.175. 1335-1340.

Day, R. H. \& Power. R. P. Frequency of apparent reversal of rotary motion in depth as a function of shape and pattern. Australian Journal of Psychology. 1963. 15. 162-174.

Day, R. H., \& Power. R. P. Apparent reversal (oscillation) of rotary motion in depth. Psychological Review. 1965. 72. $117-127$.
Grahum. C. H. On some aspects of real and apparent visual movement. Journal of the Optical Society of America. 1963, $53,1019-1025$.

Graham. C. H.. \& Gillam. B. S. Occurrence of theoretically correct responses during rotation of the Ames window. Perception \& Psych sphysics, 1970, 8, 257-260.

Hay's, W. L. Statistic's for psychologists. New York: Holt. Rinehart \& Winston, 1963.

Hershberger. W. A. Comment on "A pparent reversal (oscillation) of rotary motion in depth." Psychological Review, 1967, 74, 235-238.

Kenyon. F, C. A curious optical illusion connected with an electric fan. Science, 1898, 8. 371-372.

Leibowitz. H.. \& Moore. D. Role of changes in accommodation and convergence in perception of size. Journal of the Optical Society of America. 1966. 56, 1120-1123.

MacRae, K. D., \& Power, R. P. Real and apparent visual movement. Journal of the Optical Society of America. 1972, 62. 290.

Miles. W. Visual illusions of motion during train travel. Journal of General Psvchology, 1929, 2. 141-143.

Pastore. N. Some remarks on the Ames oscillatory effect. Psychological Review, 1952, 59, 319-323.

Power, R. P. The effect of instructions on the apparent reversal of rotary motion in depth. Quarterly Journal of Experimental Psychology, 1965, 17, 346-350.

Power, R. P. Stimulus properties which reduce apparent reversal of rotating rectangular shapes. Journal of Experimental Psychology, 1967, 73, 595-599.

Roger. R. S. Intermediate statistics. Sydney: University Co-operative Press. 1965.

Siegel. S. Nonparametric statistics. New York: McGraw-Hill. 1956.

Zegers, R. T. The reversal illusion of Ames trapezoid. Transactions of the New York Academy of Sciences, 1964. $26,377-400$.

\section{NOTES}

1. Generally, the term "stimulus" is preferred to "cue" in reference to apparent direction of movement and orientation and to depth for reasons discussed elsewhere (Day, 1972).

2. As an elliptical object rotates. its retinal projection retains its symmetry: but there is a continuous shift of its projection relative to that of its axis. For this reason, the axis of rotation must be occluded in order for complete ambiguity to obtain.

3. To establish that the occurrence of ARs for SO 3 on about half the occasions a surface passed through the frontoparallel was not coincidentally due to the particular angle subtended at the eye (see introduction), an experiment was conducted with the axis at 2.12 and $5.15 \mathrm{~m}$. For both conditions. ARs occurred on about half the occasions that the object was frontoparallel. indicating that the risual angle was less than that in which a motion perspective stimulus was operative.

(Received for publication July 7. 1972: revision received November 17.1972 .) 